\title{
Bluetongue Disease: The Growing Threat of Vector Borne Disease
}

\author{
Karam Chand* \\ ICAR-Indian Veterinary Research Institute, Mukteswar Campus, Nainital, Uttarakhand, India \\ *Corresponding Author: Karam Chand, Division of Virology, Indian Veterinary Research Institute, Mukteswar Campus, Nainital, Ut- \\ tarakhand, India.
}

Received: January 11, 2018; Published: February 01, 2018

DOI: 10.31080/ASMI.2018.01.0018

The ruminants, such as cattle, sheep and goats, contribute significantly to the agrarian economy of many countries. They play an important role in the livelihood of a large percentage of small and marginal farmers. Among various infectious diseases affecting ruminants, bluetongue (BT) is one of the major vector-borne viral disease caused by bluetongue virus (BTV). Bluetongue virus is the type species of the genus Orbivirus in the family Reoviridae. The BTV genome consists of ten linear double-stranded RNA (ds RNA) segments that code for seven structural and four non-structural proteins. BTV is transmitted between its ruminant hosts through the bite of virus-infected hematophagous Culicoides midges. To date, 27 distinct BTV serotypes have been recognized worldwide. Due to its high economic impact, BT is an Office International des Epizooties (OIE) listed disease that is strictly controlled in international commercial exchanges. Bluetongue disease was first reported in South Africa at the end of $18^{\text {th }}$ century. The disease was initially called "fever" or "epizootic catarrh", but was later referred to as "malarial catarrhal fever of sheep". The disease was later renamed "bluetongue" with reference to the characteristic cyanotic tongues that were occasionally observed in infected sheep. Bluetongue outbreaks correlate with climatic conditions, the density of sheep population and breeds reared in different geographical region. The disease is endemic in tropic and subtropics climates, but due to global warming vector has crossed the geographical range. Now, the disease outbreak is reported in northwest Europe showing the changing pattern of disease. The ability of Culicoides midges to transmit the BTV is influenced by ambient temperature, humidity and seasonal rainfall. Variation in ambient temperatures, humidity, water logging etc. affect the breeding condition for the vectors and correlates with disease outbreak in the different geographical region. All ruminants are susceptible to BTV infection, but clinical disease is observed mainly in sheep. However, in Western and Central Europe even cattle showed clinical disease in an outbreak caused by BTV-8 serotype. Bluetongue in sheep is manifested by fever, hyperemia of lips and nostrils, salivation, nasal discharge, ulcer on oral mucosa, edema of the tongue and lips. Cyanosis of the tongue is observed in rare cases. At the end of the fever, affected sheep have coronitis, laminitis, stand with an arched back and reluctant to move. The serotypes of BTV exist in a complex network of serological cross relationship, varying from partial to no protection between heterologous strains, posing a great challenge to control the disease. Intermixing of animals through inter- boundary movement and spread of competent vectors to different regions complicate the problem further. The BTV can be isolated in embryonated chicken egg (ECE), cell culture or in sheep. The identification of BTV in blood or tissue sample is done by reverse transcription-polymerase chain reaction (RT-PCR), real-time RTPCR, virus neutralization test (VNT) and antigen-capture ELISA. The serogroup-specific antibody against BTV can be detected by agar-gel immunodiffusion test, serum neutralization test (SNT), competitive and indirect-ELISA. There is no specific treatment for bluetongue disease. For effective control of BT, it is important to commence sentinel, vector trap systems, checks on animal movement, studies on Culicoides vectors, continuous disease surveillance, zone-specific multivalent vaccines and proper diagnosis of disease. For prophylactic immunization, live attenuated, inactivated and new generation vaccine like recombinant vector vaccine, subunit vaccines, virus-like particles (VLPs) are being developed. Live attenuated vaccines, as well as inactivated vaccines have been successfully used in China, South Africa, Europe and other countries. To monitor the serotypes circulating in the different parts, it is necessary to set up a system so that particular serotype of the virus can be identified and included in the multivalent vaccine formulation for successful control of the disease. It is difficult to completely eliminate the Culicoides midges from the natural environment; however, it can be reduced up to ineffective level by using approved insecticides.

\section{Volume 1 Issue 3 March 2018 \\ (C) All rights are reserved by Karam Chand.}

